\begin{tabular}{|l|l|l|l|l|l|}
\hline Cuadernos I. Geográfica & 16 & $1-2$ & $7-30$ & Logroño & 1990 \\
\hline
\end{tabular}

\title{
DISTRIBUCION SUPERFICIAL DEL TAMAÑO DE LOS SEDIMENTOS EN DOS CONOS ALUVIALES GENERA- DOS POR UNA TORMENTA (ALTO VALLE DEL NAJERILLA)
}

Amelia GOMEZ-VILLAR*

\begin{abstract}
RESUMEN. -En este artículo se estudia la distribución espacial de los sedimentos en los conos de Pitare y Banadero (Alto valle del Najerilla) atendiendo a las caracteristicas de los cantos medidos en 18 puntos de su superficie. Los resultados muestran que la organización superficial del eje mayor y tamaño medio de los cantos no guarda relación con la distancia desde el ápice a la base y con la pendiente dentro del cono y su evolución está en función de los procesos deposicionales que formaron ambos conos.

SUMMARY. -Superficial distribution of sediment size in two alluvial fans triggered by a rainstorm (Najerilla High Valley). In this paper the spatial distribution of the sediments is studied in the alluvial fans of Pirare and Banadero (Najerilla High Valley, Iberial System, Spain), according to the characteristics of gravels measured in 18 points. The results show that the superficial organization of the A-axis and of the mean size of the gravels has no relation with the distance from the apex and with the slope. Their evolution is a function of depositional processes that buildt such alluvial fans.
\end{abstract}

Palabras clave: Conos aluviales, sedimentos, Sierra de la Demanda (Sistema Ibérico).

Key words: Alluvial fans, gravels, Sierra de la Demanda (Iberian System).

Los efectos geomorfológicos y sedimentarios sobre conos aluviales causados por fenómenos excepcionales -que juegan un papel muy significativo en la

*Dpto. de Geografia y Ordenación del Territorio. Coleglo Universitarło de La Rloja, Logroño. 
movilización de sedimentos en el área fuente (KELSEY, 1980)- son comunes en regiones con distintos ambientes climáticos. WELLS \& HARVEY (1987) mostraron que en regiones templadas y húmedas, ocasionalmente, sucesos de poca frecuencia y alta magnitud pueden ser importantes en la modificación del paisaje, en contraste con el principio de WOLMAN \& MILLER (1960), según el cual las alteraciones que sufre el paisaje están más estrechamente relacionadas con sucesos geomorfológicos de frecuencia e intensidad moderada -con un periodo de retorno de menos de 1 año a varios años-. Para muchos autores, la formación de conos aluviales y/o su alteración vienen a ser, en su mayor parte, el resultado de fuertes tormentas de corta duración (BEATY, 1974) y señalan que la descarga total de sedimentos es equivalente al valor de la descarga realizada por procesos que ocurren más frecuentemente durante muchos años de actividad (PIERSON. 1980).

Las cabeceras de barrancos y laderas de pequeñas cuencas en zonas montañosas son las principales áreas fuente de sedimentos de los conos (CoSTA \& JARRET, 1981; Iso et al, 1970). Después de una intensa precipitación el equilibrio existente en el sistema cuenca/cauce es alterado y varian las condiciones de sedimentación a las que el sistema fluvial se había adaptado. Este es el caso de los Barrancos Pitare y Banadero, en el alto valle del Najerilla -Sierra de la Demanda (Sistema Ibérico)-. Sus cauces, tras una fuerte tormenta, muy local, experimentaron un brusco aumento de caudal y transportaron grandes cantidades de sedimentos desde la cabecera depositándolos en forma de conos, por medio de coladas de piedras y procesos fluviales (ver GARCIA-RUIZ et al, 1988), al alcanzar el rio principal y represando sus aguas en ambos casos.

Muchos trabajos se han escrito relacionados con la formación de conos aluviales como respuesta geomorfológica a este tipo de sucesos (BAKER, 1977; CAINE, 1980; HARVEY, 1984, 1986; NEWSON, 1980) y sobre sus caracteristicas sedimentológicas (GARCIA-RUIZ et al, 1988; PIERSON, 1980; TUNBRIDGE, 1983; WELLS \& HARVEY, 1987), para regiones áridas y semiáridas y zonas templadohúmedas. El objetivo de este trabajo es estudiar las características de la distribución espacial del tamaño de los sedimentos en los depósitos superficiales y canales de los conos de Pitare y Banadero y observar los cambios que se producen en su evolución desde el ápice a la base.

\section{Area de estudio}

Los Barrancos de Pitare y Banadero se localizan en el alto valle del Najerilla: el primero vierte sus aguas directamente a este rio y el segundo se halla en la margén derecha del Tobía, afluente del Najerilla aguas arriba de la localidad de Bobadilla (Figura 1). Sus cuencas, muy pequeñas $-0,76 \mathrm{~km}^{2}$ la de Banadero y $2,125 \mathrm{~km}^{2}$ la de Pitare (Tabla I)- y de vertientes muy pronunciadas -pendientes superiores al $45 \%$ - se inscriben en el secrtor oriental del macizo paleozoico de la Sierra de la Demanda. Se trata de un conjunto montañoso que corresponde al sector más noroccidental del Sistema Ibérico, caracterizado por una linea de 
cumbres muy homogénea -en torno a los $2.000 \mathrm{~m}$ de altitud-, a partir de las cuales descienden unas laderas con fuertes pendientes como consecuencia del encajamiento de la red fluvial (ARNAEZ, 1987; CALVO, 1975).

La litologia de estas cuencas se compone de cuarcitas (Banadero) y una alternancia de cuarcitas y pizarras (Pitare). El ambiente climático está condicionado por la posición avanzada de la Sierra hacia el W. Los flujos del NW, oceánicos, descargan su húmedad mediante precipitaciones (ARNAEZ, 1984) que superan los 1.000 litros a partir de los $1.500 \mathrm{~m}$. de altitud, con un máximo invernal y mínimo estival (ARNAEZ y GaRCíA-RUIZ, 1984).

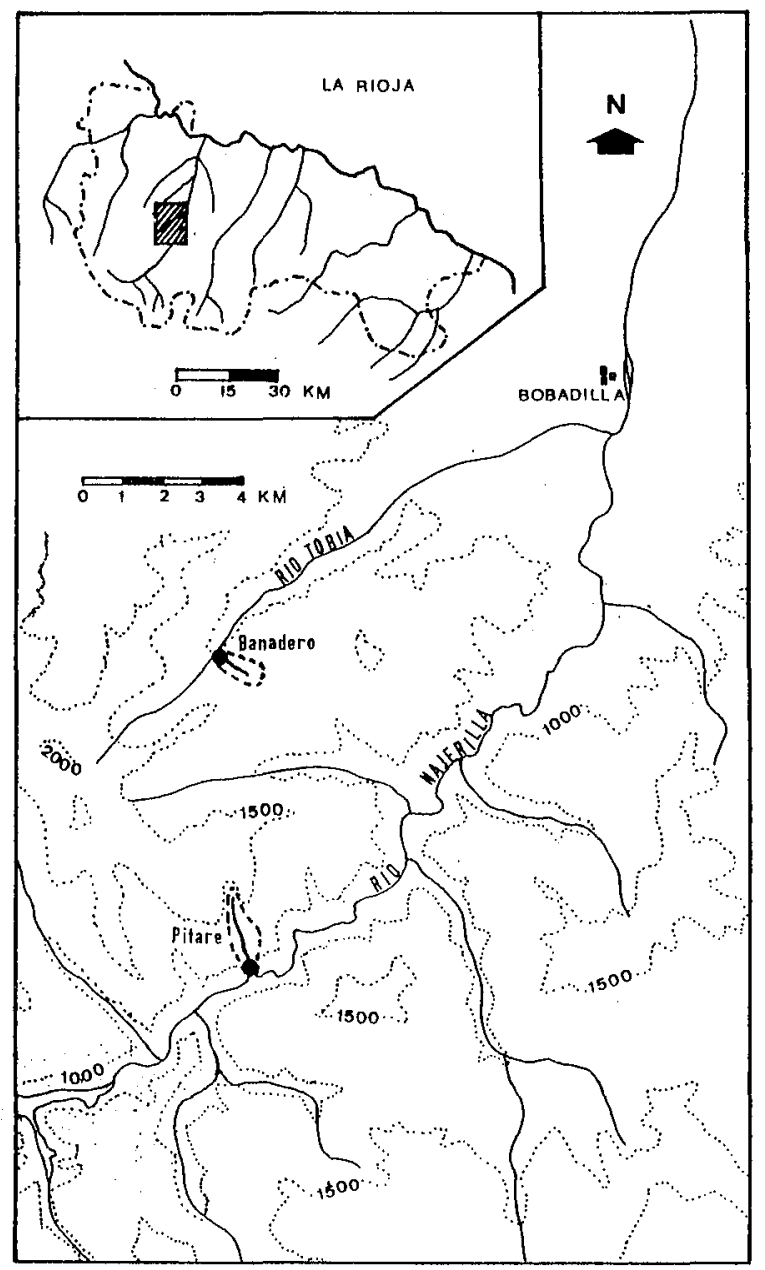

Fig.1. Localización de los conos Pitare y Banadero.

La actividad de estos barrancos -con unas pendientes medias que rondan el $30 \%$ y unas laderas cubiertas por un denso bosque de hayas en la cuenca del Banadero y matorral con ejemplares dispersos de Quercus pyrenaica y Quercus ilex rotundifolia en el Barranco de Pitare-, condicionada por la localización en sus cuencas de recepción de una cabecera activa (Banadero) y desprendimien- 
tos (Pitare), era prácticamente nula con pocos aportes de sedimentos y cauces colonizados por la vegetación, tal como revelan las fotografias aéreas de 1978 . $\mathrm{Su}$ reactivación se produjo tras una tormenta, de carácter muy puntual, en la vertiente suroriental del macizo de San Lorenzo, en un ambiente de montaña calificado como estable por ARNAEZ (1987) y coincidió con el paso de un frente frio que afectó también a otros puntos del Sistema Ibérico, descargando tormentas aisladas. Lamentablemente no existen registros pluviométricos fiables; en la estación más próxima al sector afectado, tan sólo se recogieron 13 mm. el día de máxima precipitación, pero indudablemente la lluvia caîda en algunos puntos fue muy superior (ARNAEz et al, 1987). Este suceso dió lugar a la producción, movilización y descarga de gran cantidad de material y a formación de pequeños conos aluviales en sus desembocaduras, muy empinados y de aspecto muy torrencial, incididos posteriormente por flujos de agua. La profunda disección del cono de Pitare en el sector central y distal (Foto 1) esta asociada a su destrucción (WASSON, 1977). La formación de este cono estuvo propiciada por un incendio forestal declarado en esa zona en fechas anteriores a la tormenta. La destrucción parcial del bosque tras el incendio dejó muy desprotegidas las laderas y alteró profundamente su dinámica geomorfológica (GARCÍA-Ruiz et al, 1988), desencadenando, además, en todo el sector, numerosos movimientos en masa.

TABLA 1

Algunos aspectos morfométricos de las cuencas y sus conos.

\begin{tabular}{l|c|c}
\hline & Banadero & Pitare \\
\hline Tamaño cuenca $\left(\mathrm{km}^{2}\right)$ & 0.76 & 2.125 \\
Altitud media & 1312 & 1175 \\
Pendiente media (\%) & 46 & 47.46 \\
Orientación dominante & $\mathrm{W}$ & $\mathrm{S} \mathrm{y} \mathrm{E}$ \\
Densidad de drenaje & 2.7 & 3.7 \\
Pendiente media barranco & 33 & 29.6 \\
Area del cono & $<1 \mathrm{ha}$ & $<1 \mathrm{ha}$ \\
Pendiente media $\left({ }^{\circ} \mathrm{C}\right)$ & $8^{\circ}$ & $8.5^{\circ}$ \\
\hline
\end{tabular}

\section{Métodos}

Para la elaboración de este trabajo se ha aplicado una metodología sencilla basada en la recogida de datos en los dos conos seleccionados:

-En una primera fase se han efectuado, con pantómetros de $25 \mathrm{~cm}$. y $1 \mathrm{~m}$., varios perfiles topográficos transversales en la zona apical, media y distal de ambos conos que nos han permitido observar algunas caracteristicas de la morfologia superficial del cono y las incisiones hechas por el flujo durante y posteriormente a la formación de los conos.

-Por otro lado, se establecieron 18 puntos de muestreo en cada cono; sobre 


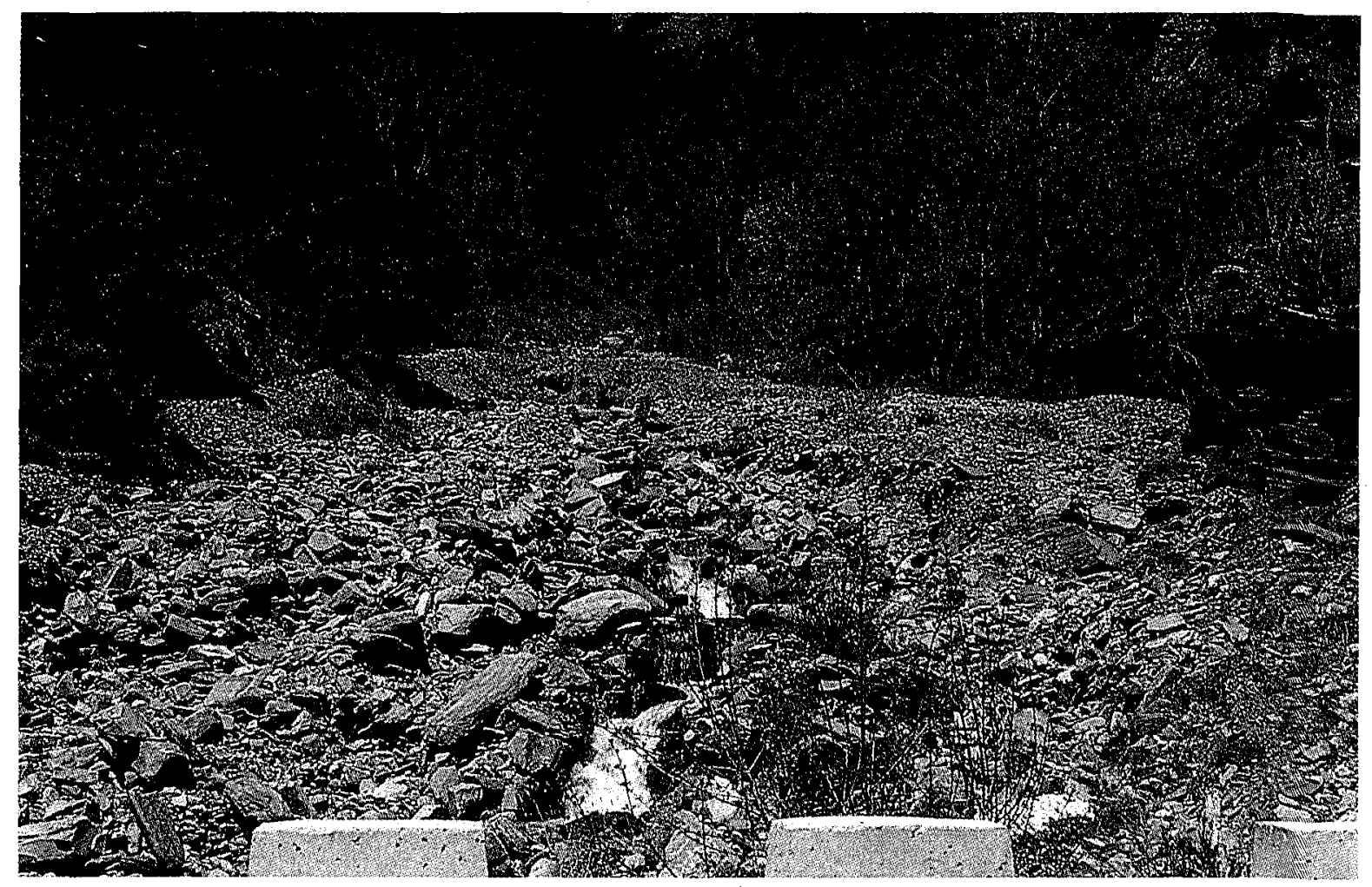

Foto 1. Cono de Ptare. Profunda disección en los sectores central y distal.

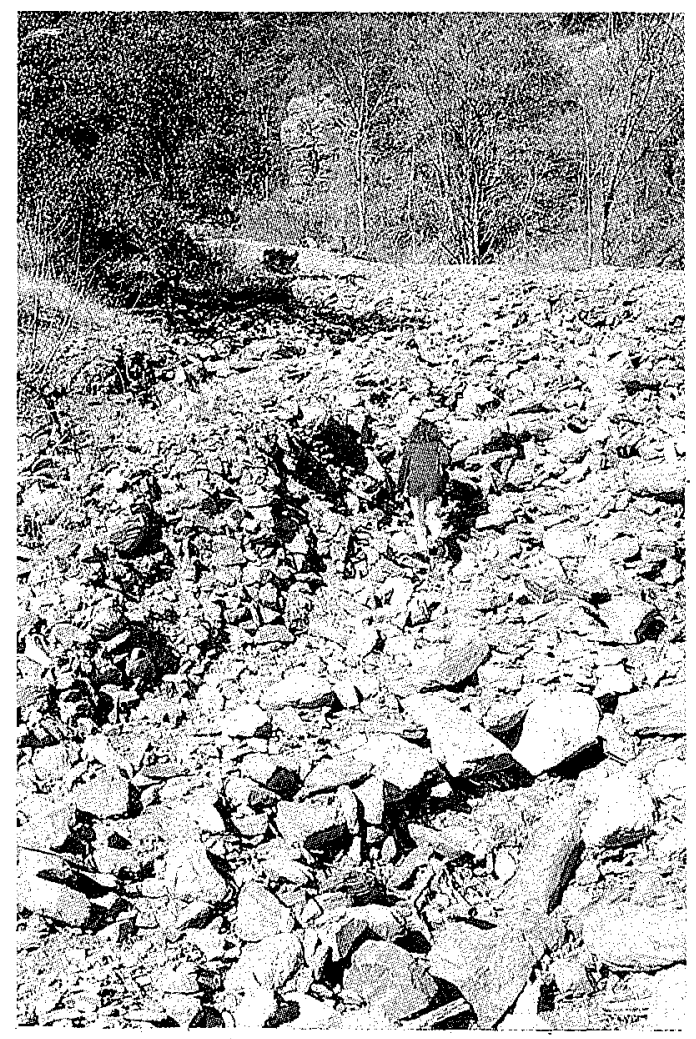

Foto 2. Sedimentos gruesos en los sectores central y distal del cono de Pitare, formados por coladas. 
ellos se hicieron descripciones cualitativas de campo y se tomaron datos morfométricos del eje $a$ y del eje $b$ de 50 cantos, escogidos al azar según el método de Wolman (1954), asî como los ejes de los 10 cantos más grandes encontrados en cada estación. A la vez se tomó la pendiente longitudinal en cada uno de los puntos muestreados.

Asimismo, en tres perfiles longitudinales radiales se ha medido el eje mayor (a) de los cantos cada $50 \mathrm{~cm}$., desde el apice a la base.

El tratamiento estadístico posterior, para caracterizar globalmente el conjunto de las muestras y analizar las diferencias más notables en los distintos ambientes deposicionales, reveló un comportamiento y evolución similar de ambos ejes por lo que se trabajó con el eje longitudinal (a) que representa bien la competencia del transporte con la distancia (BOOTHROYD \& NUMMEDAL, 1978; BlissenBACH, 1952).

\section{Resultados}

La elaboración y análisis de los datos morfométricos nos permite conocer algunos de los aspectos de las condiciones hidrodinámicas en que fueron depositados los conos de Pitare y Banadero. Las Figuras 2 y 3 representan los histogramas de frecuencias del tamaño de los sedimentos de cada cono y revelan la heterogeneidad de su distribución en toda la superficie del cono. En los perfiles transversales se señala la localización de algunos de los puntos muestreados y en la Tabla II se indican, además, otras características de las muestras llevadas a cabo:

Cono de Pitare. Podemos distinguir varios hechos:

a) El mayor número de intervalos de frecuencias se da en el canal funcional actual (puntos 4, 6, 10 y 16) y en toda la zona distal central (puntos 14 y 15), es decir, en los sectores más disectados del cono (ver Figura $4 \mathrm{Ay} \mathrm{C}$ ). En el canal, en la zona apical, el tamaño medio de los cantos es de $148.6 \mathrm{~mm}$. y va aumentando progresivamente con la distancia hasta $246.04 \mathrm{~mm}$. en el punto 16. En estos puntos, casi el $30 \%$ de los clastos tiene tamaños superiores a 200 $\mathrm{mm}$. siendo prácticamente inexistentes los inferiores a $25 \mathrm{~mm}$. Los cantos con ejes inferiores a $100 \mathrm{~mm}$. suponen un porcentaje importante (en torno al 35\%). La enorme dispersión está en función de las características y funcionamiento del propio canal, muy empinado y con poca capacidad de selección del material en la escasa distancia recorrida desde el ápice a la base. En la zona distal, el calibre medio de los cantos también es importante $(165.3$ y $202.5 \mathrm{~mm}$. respectivamente) pero con una distribución más regular de los intervalos. Los valores de la desviación estandar y coeficiente de variación (Tabla II), los más elevados de todos los puntos muestreados, confirman este hecho. 


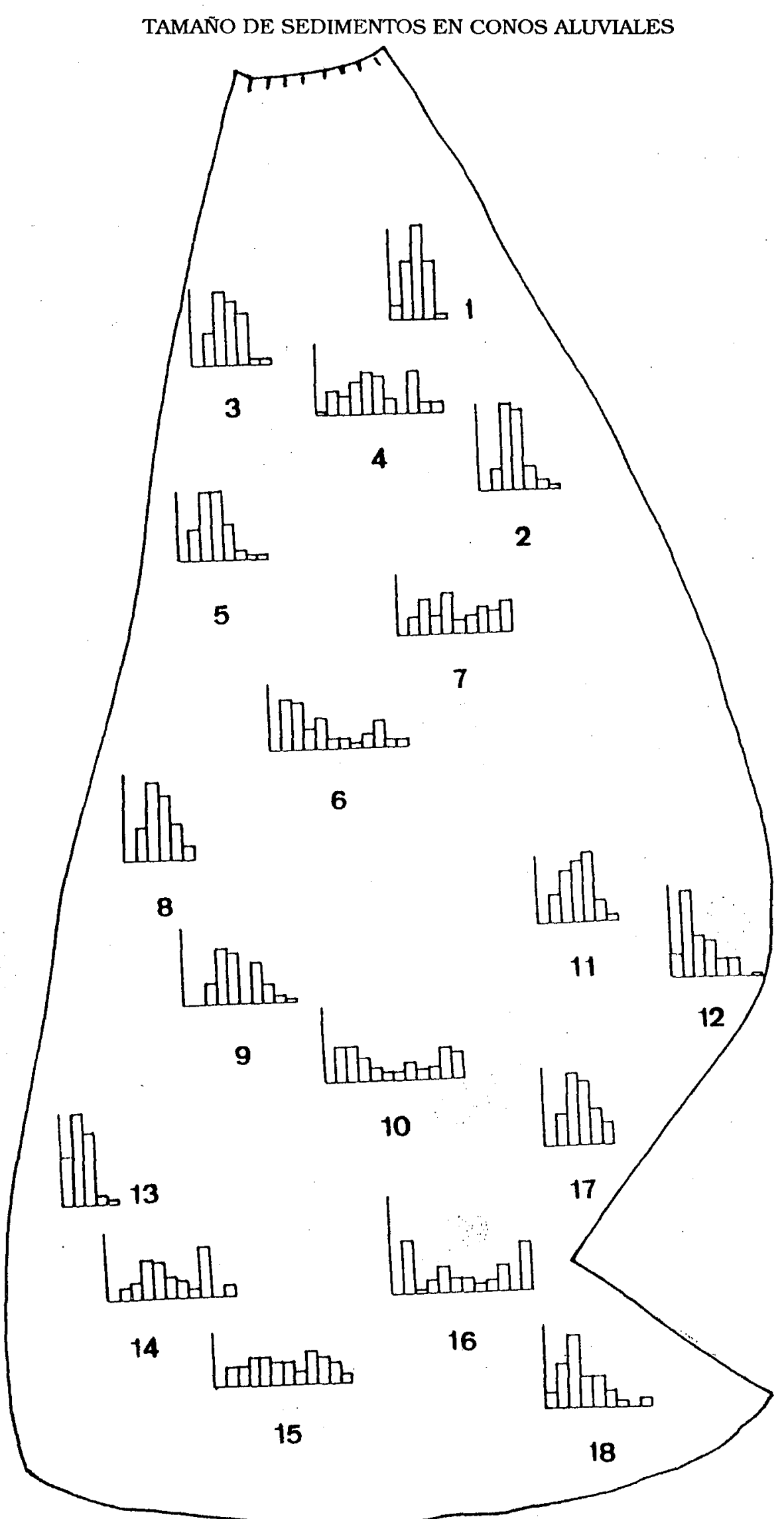

Fig. 2. Distribuclón de los histogramas de frecuenclas del tamaño de cantos en el cono de Pitare. 


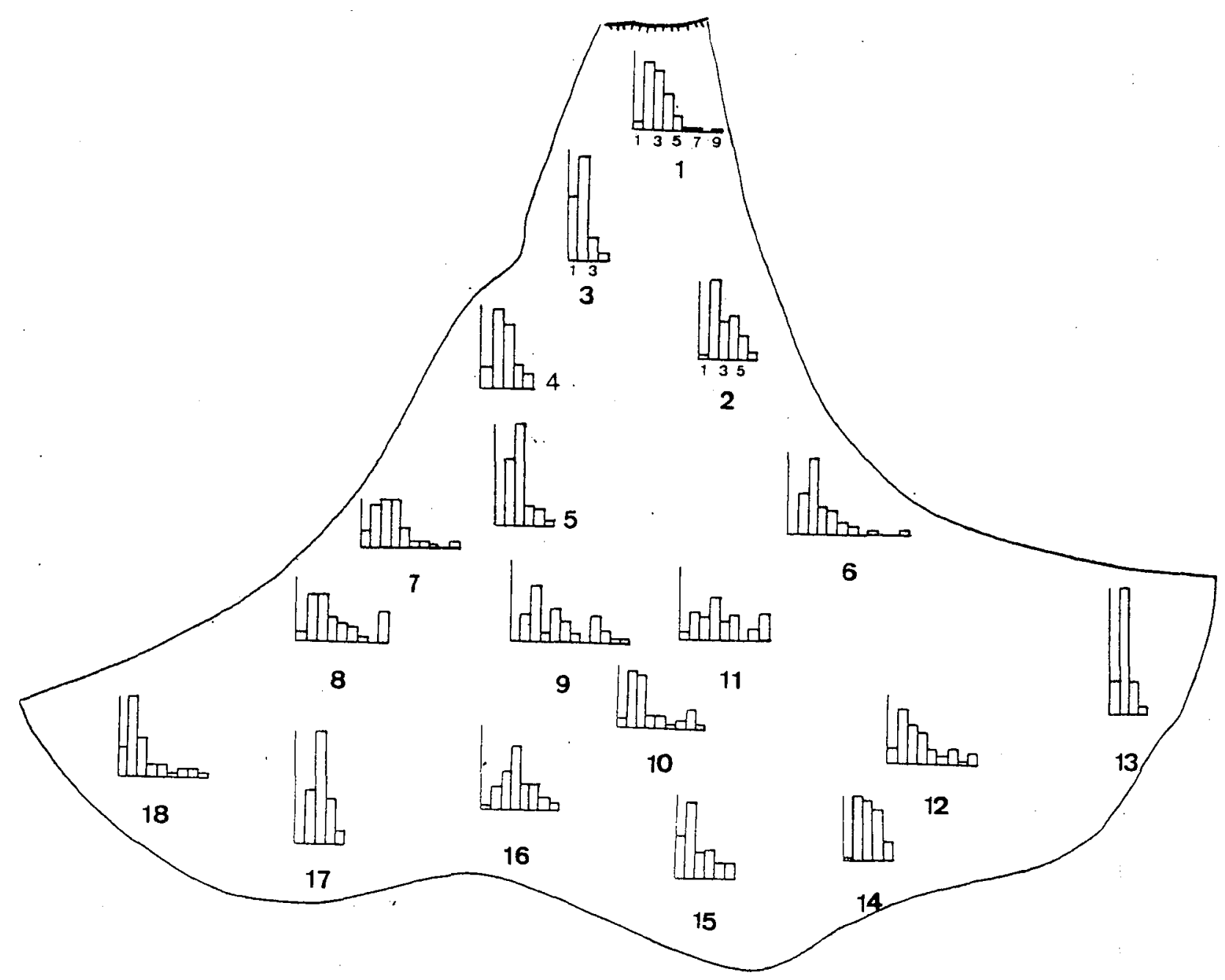

Fig. 3. Distribuclón de los histogramas de frecuencias del tamaño de los cantos del cono de Banadero.

b) El material de los niveles más superficiales del cono, no disectados por flujos posteriores a la deposición (Figura 4) presenta una distribución más homogénea (puntos $3,5,8$ y 13, en el sector derecho y puntos 1,2 y 12 en el izquierdo). Los histogramas se estrechan notablemente y muestran una concentración muy importante de los tamaños de los sedimentos en las clases comprendidas entre 25 y $100 \mathrm{~mm}$. (<60\%) y no hay cantos más grandes de 200 $\mathrm{mm}$. Los valores de las medidas de dispersión del calibre de los cantos son poco significativas en relación al total de los puntos muestreados. La mayor homometria de los sedimentos se da especialmente en el sector derecho, aunque en ambos sectores se aprecia una ligera tendencia a la disminución del tamaño medio de los cantos hacia la base del cono. Este comportamiento es lógico si tenemos en cuenta que forman parte de un ambiente deposicional que corresponde a los últimos pulsos de la formación del cono, con flujos menos violentos que en la primera fase de deposición. 
TAMAÑO DE SEDIMENTOS EN CONOS ALUVIALES

TABLA 2

Caracteristicas del tamaño de los cantos en los puntos muestreados

\begin{tabular}{rrrrr} 
& Media & Desu.stand & Coef.var. & Pend. ${ }^{\circ}$ C) \\
\hline Ba01 & 66.70 & 38.75 & 58.09 & 7 \\
Ba02 & 66.04 & 31.38 & 47.51 & 7 \\
Ba03 & 35.18 & 15.72 & 44.68 & 6 \\
Ba04 & 54.14 & 23.48 & 43.37 & 6 \\
Ba05 & 63.70 & 22.67 & 35.58 & 6 \\
Ba06 & 88.50 & 77.95 & 88.08 & 5 \\
Ba07 & 84.46 & 67.04 & 79.38 & 7 \\
Ba08 & 93.98 & 63.88 & 67.97 & 8 \\
Ba09 & 143.56 & 124.41 & 86.66 & 9 \\
Ba10 & 84.08 & 52.00 & 61.85 & 7 \\
Ba11 & 116.00 & 81.30 & 70.09 & 5 \\
Ba12 & 85.04 & 57.97 & 68.17 & 7 \\
Ba13 & 39.48 & 15.51 & 39.28 & 7 \\
Ba14 & 65.18 & 27.01 & 41.45 & 9 \\
Ba15 & 59.72 & 37.38 & 62.60 & 7 \\
Ba16 & 93.10 & 40.90 & 43.93 & 7.5 \\
Ba17 & 66.04 & 21.64 & 32.78 & 8 \\
Ba18 & 65.70 & 52.74 & 80.28 & 9 \\
& & & & \\
Pi01 & 78.40 & 25.00 & 31.89 & 9 \\
P102 & 62.96 & 20.55 & 32.65 & 10 \\
Pi03 & 81.44 & 30.07 & 36.93 & 9 \\
Pi04 & 148.60 & 98.10 & 66.02 & 6 \\
Pi05 & 82.38 & 33.75 & 40.95 & 11 \\
Pi06 & 160.86 & 144.82 & 90.03 & 6 \\
Pi07 & 160.20 & 97.57 & 60.90 & 9 \\
P108 & 78.02 & 27.20 & 34.86 & 7 \\
Pi09 & 131.08 & 54.50 & 41.58 & 9 \\
Pi10 & 227.54 & 200.80 & 88.25 & 6 \\
P111 & 89.44 & 29.00 & 34.35 & 8 \\
Pi12 & 66.14 & 39.51 & 59.74 & 7 \\
Pi13 & 45.92 & 22.30 & 48.57 & 12 \\
P114 & 165.32 & 102.92 & 62.25 & 11 \\
P115 & 205.56 & 154.39 & 75.11 & 14 \\
P116 & 246.04 & 214.04 & 84.00 & 12 \\
Pi17 & 83.72 & 28.63 & 34.20 & 9 \\
Pi18 & 48.33 & 59.11 & 9 \\
\hline & & & & \\
\hline
\end{tabular}

c) La tendencia indicada más arriba, aunque con tamaños medios superiores, se cumple en las estaciones localizadas en el sector central, en la zona media y distal del cono, en niveles intermedios entre los dos anteriores, con una disminución bien marcada del tamaño de los cantos hacia las partes más alejadas del ápice $(160.2,131.68,89.44$ y $81.46 \mathrm{~mm}$. respectivamente). La 
distribución de las frecuencias es la misma en los puntos 11 y 17 -prácticamente la totalidad de los cantos muestreados tienen valores entre 25 y $125 \mathrm{~mm}$. Los intervalos se estiran hacia clases más elevadas en los puntos $18 \mathrm{y}$, sobre todo, 7 , aunque el $80 \%$ de los sedimentos tiene tamaños inferiores a $200 \mathrm{~mm}$. El punto 9 en la zona media enlaza con el nivel de los puntos 14 y 15, pero se acorta el número de intervalos y desciende el tamaño medio: $131.12 \mathrm{~mm}$.
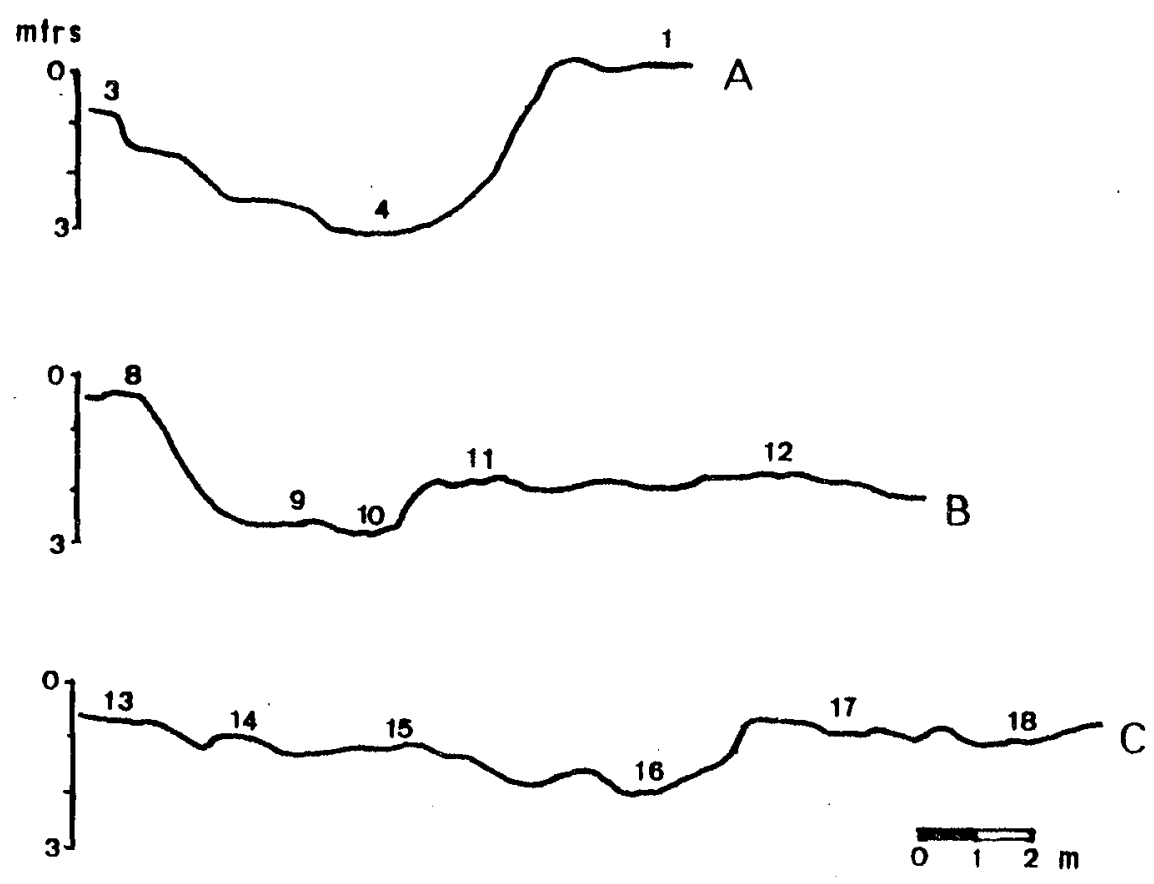

Fig. 4. Perfiles topográflcos transversales en la zona apical, media y distal en el cono de Pitare.

Cono de Banadero. Los procesos que han intervenido en su formación quedan reflejados en las características de los datos morfométricos, que ofrecen una mayor complejidad en este cono:

a) La mayor amplitud de rangos de tamaños se da en el sector central. Aquí se muestrearon varios puntos correspondientes a un sistema deposicional a modo de coladas de piedras. Si observamos el perfil B de la Figura 5 se pueden apreciar las márgenes bien definidas de dos coladas (puntos 9 y 11). Los sedimentos de estos depósitos son heterométricos, con un gran número de intervalos de frecuencias y los tamaños medios de cantos más elevados de todos los sectores del cono (143.56 y $116 \mathrm{~mm}$ ). Los puntos 7 y 10 pertenecen a áreas más disectadas dentro de este conjunto, con cantos más pequeños que los anteriores. Todos los histogramas presentan una tendencia bimodal, concentrándose más de la mitad del volumen de cantos muestreados en los intervalos de 25 a $100 \mathrm{~mm}$. y de más de $200 \mathrm{~mm}$. La desviación estandar del calibre de los cantos tiene valores muy superiores al del resto de las estaciones muestreadas.

b) En torno al ápice, fuertemente incidido (perfil A de la Figura 5) se llevaron 
a cabo tres muestreos: los puntos 3,4 y 5 , que corresponden a niveles superio res del último estadio de deposición del cono. En los histogramas se ha acortado la gama de clases por ausencia de cantos superiores a $150 \mathrm{~mm}$. y adquieren importancia los datos situados entre las frecuencias de 0 y $75 \mathrm{~mm}$. (más del $80 \%$ del total de los clastos se incluye en esos intervalos). El punto 13, en la zona distal izquierda, presenta las mismas características de distribución del calibre de los sedimentos, e incluso más acentuadas: el $90 \%$ de los mismos tiene un eje inferior a $75 \mathrm{~mm}$. Así, el tamaño medio de los cantos ha descendido visiblemente en todos los puntos, al igual que los valores de las medidas de dispersión. mlrs
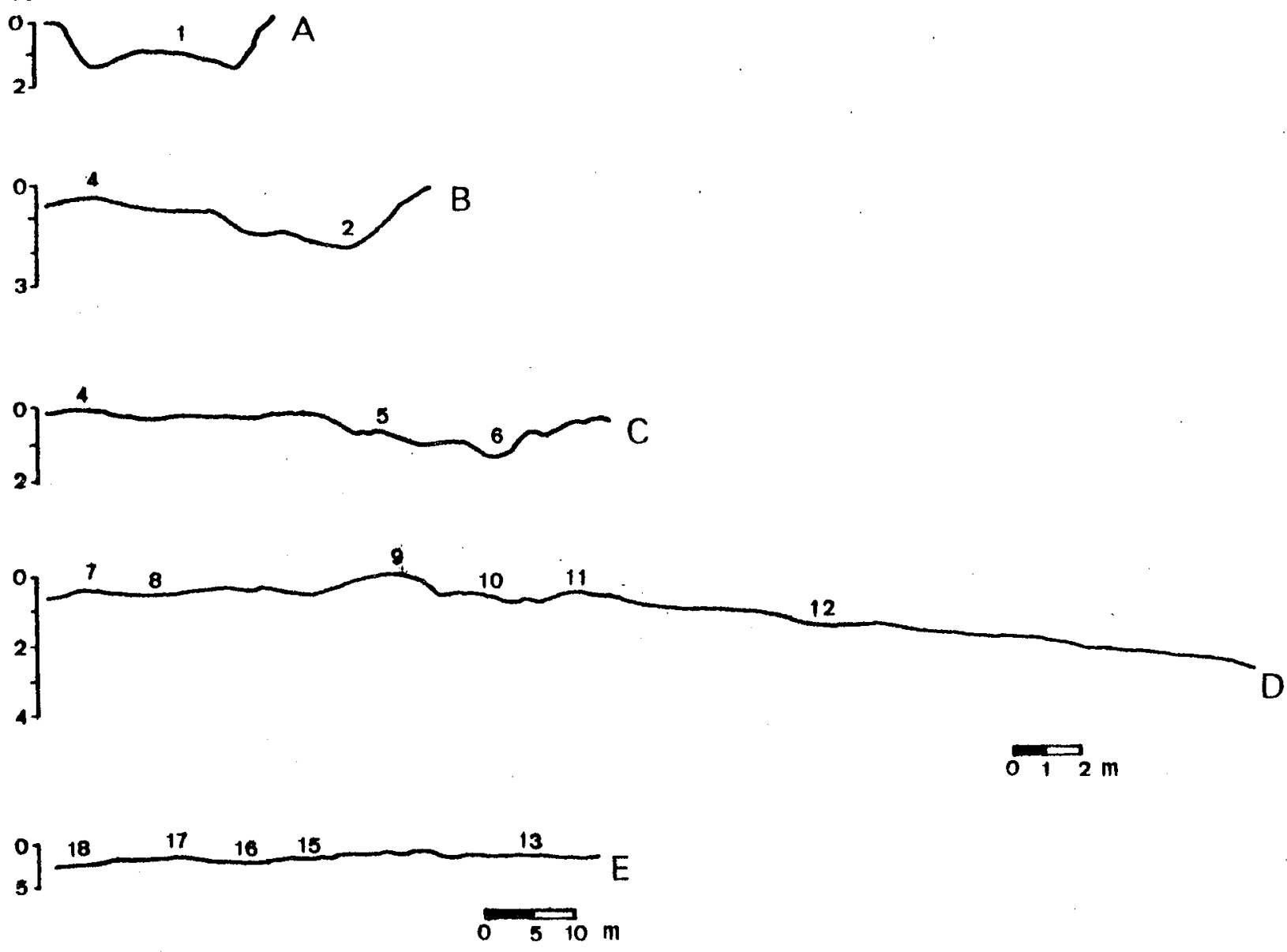

Flg. 5. Perfles topográficos transversales en el cono de Banadero.

c) La zona distal está formada también por coladas de menor relieve que en la zona media e incididas por numerosos canales (perfil C, Figura 5). El punto 16, más disectado, es el que engloba un mayor número de frecuencias, con cantos inferiores a $25 \mathrm{~mm}$. (entre 5 y 15\%) y bloques de mayor tamaño y presenta los valores más altos de dispersión, pero no así del tamaño medio -65.3 milímetros.-, que corresponde a los puntos muestreados en la superficie de las coladas menos afectadas por flujos posteriores. En estos puntos -14, 15 y 17casi el 90\% de los cantos se incluye entre las clases de 25-125 mm. 
d) Un mayor número de intervalos se da en el canal actual funcional - puntos $1,2,6$ y 12 . Más del $65 \%$ de los sedimentos tiene un calibre inferior a $100 \mathrm{~mm}$, pero en los puntos, 1, 6 y 12 encontramos cantos de más de $200 \mathrm{~mm}(<6 \%)$. En el punto 1 se trata de cantos caídos inmediatamente aguas abajo de la cascada y que el flujo posterior ha sido incapaz de remover. En el caso de los puntos 6 y 12 son bloques retomados de las coladas. El tamaño medio se halla muy matizado por la presencia de estos clastos más gruesos. Los puntos 8 y 18, que corresponden a un canal abandonado presentan las mismas caracteristicas en su evolución, aunque con valores más bajos para el conjunto de las dos muestras.

En las Figuras 6 y 7 se muestra la progresiva evolución del tamaño de los cantos cada $50 \mathrm{~cm}$. desde el ápice a la base a lo largo de tres perfiles radiales longitudinales. Las imágenes que presentan son muy expresivas de las irregularidades que se producen dentro de esa evolución:

Cono de Pitare, en el sector derecho (Figura 6 A) no se aprecian alteraciones significativas. Todos los cantos se hallan por debajo de los $200 \mathrm{~mm}$., y no hay clastos de tipo gravilla -inferiores a $25 \mathrm{~mm}$.-. Se trata de un material muy homométrico, que disminuye ligeramente de tamaño hacia la parte distal del
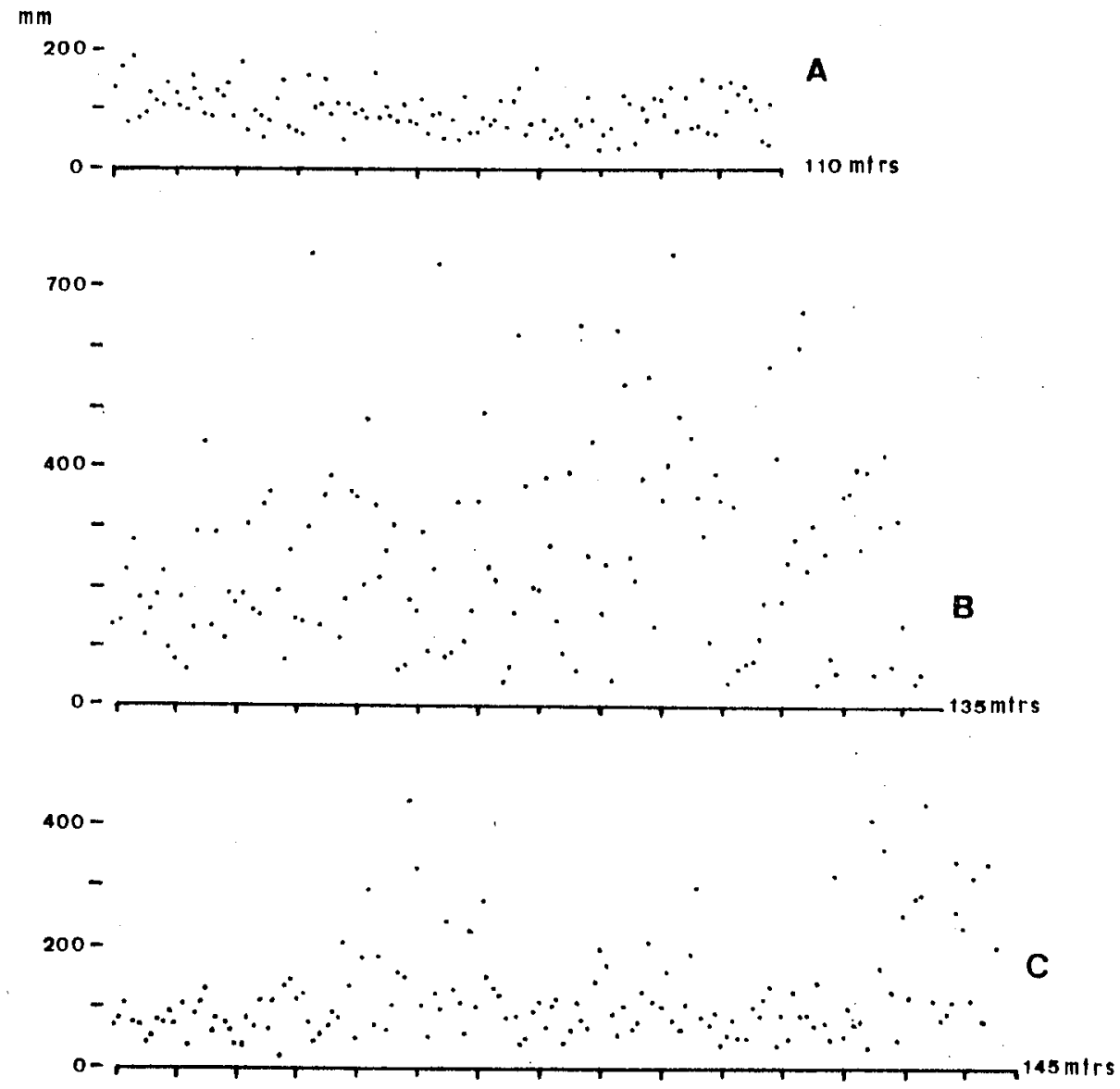

Fig. 6. Evoluclón del tamaño de cantos en el cono de Pitare desde el áplce a la base. A. Sector "derecho". B. Sector central. C. Sector "izquierdo". 
cono y que ha sido depositado en la fase final de la formación del cono, con material más seleccionado. Sin embargo, en el perfil correspondiente al canal funcional actual, en la zona más profundamente incidida, la anterior tendencia varia bruscamente mostrando la gran dispersión que existe en este sector, encontrándose gravas inferiores a $25 \mathrm{~mm}$. y una proporción importante de bloques superiores a $400 \mathrm{~mm}$. de diámetro. Se trata de un canal muy empinado (Tabla II) con una morfologia a base de riffles, formados por los cantos más grandes, y pools, donde se acumula material más fino. La tendencia es más acusada en la parte distal del cono, totalmente disectada. El gráfico $6 \mathrm{C}$ presenta unas características intermedias entre las dos anteriores: sobre una base de cantos pequeños ( $<100 \mathrm{~mm}$.) que disminuyen en número hacia la base, se producen tres picos formados por cantos de calibre muy superior, de hasta $\mathbf{4 5 0}$ $\mathrm{mm}$. Se trata de un sector, localizado en una zona intermedia entre el canal y el sector de la margen izquierda, compuesto por coladas lobuladas de escaso relieve, que se superponen y entrecruzan y dejan entre si pequeños escalones que coinciden con los frentes de las coladas y que tienen material de calibre superior, representado por esos picos más acentuados.

Cono de Banadero. La evolución del calibre de los cantos varía sensiblemente en este cono (figura 7). En los perfiles 7 A y B (sector central y derecho)
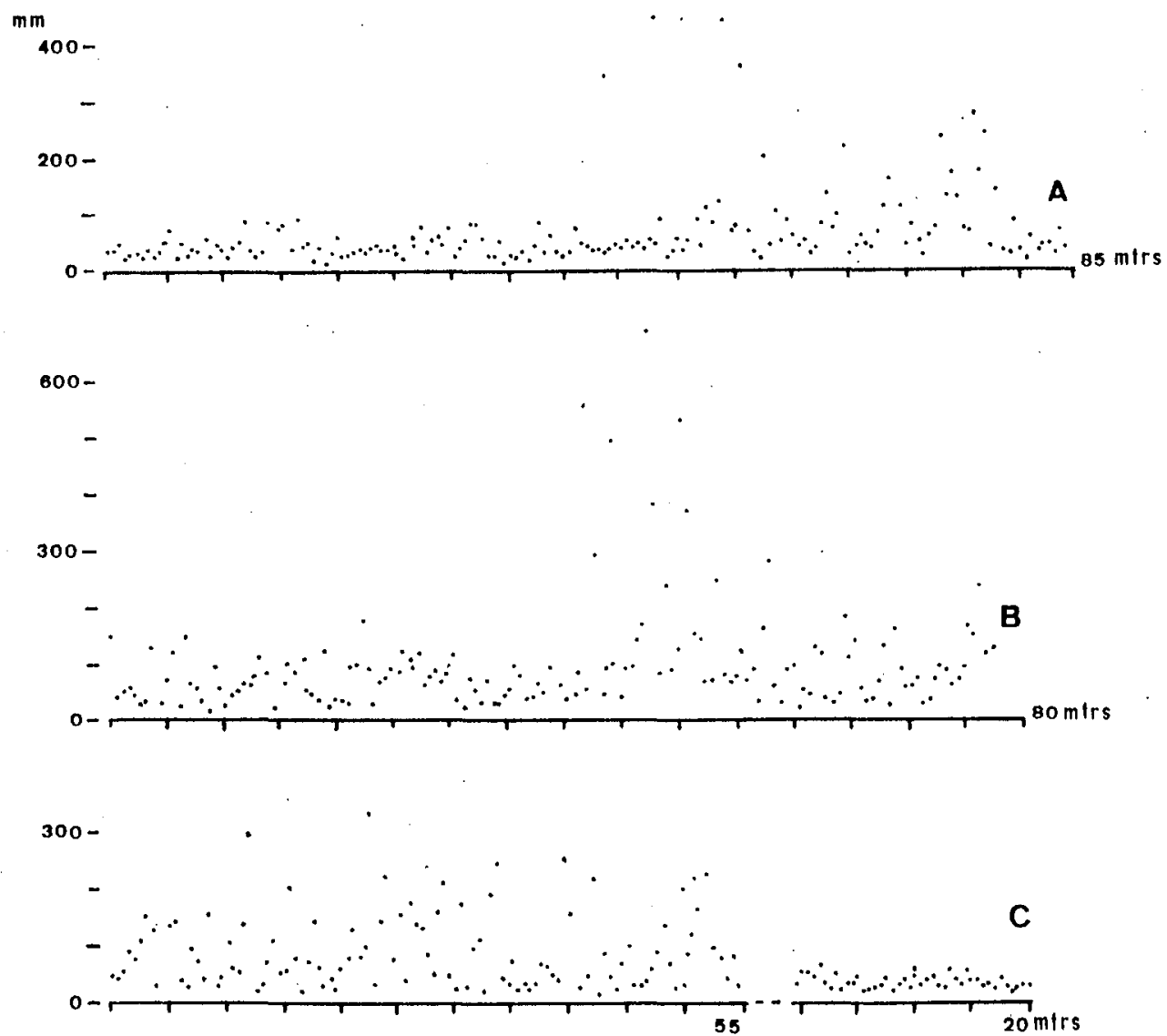

Fig. 7. Evolución del tamaño de cantos en el cono de Banadero. A. Sector "derecho". B. Sector "central". C. Sector "izquierdo". 
encontramos una doble tendencia: 1) los primeros metros hasta la zona media se caracterizan por la homometría del depósito -tamaños inferiores a $100 \mathrm{~mm}$., mejor marcados en el perfil A-; se trata de la superficie no alterada del cono, con un material bien clasificado, depositado mediante procesos fluviales, en los últimos pulsos de expansión del flujo de sedimentos. Inmediatamente cono abajo esta tendencia se rompe y desaparece gran parte de la base de gravas, dominando cantos de gran tamaño $(400 \mathrm{~mm})$. Corresponde al sector medio del cono, depositado mediante flujos de piedras, retomadas de aguas arriba, en el ápice, donde se encuentra una pequeña cascada, que favoreció la acumulación a sus pies de estos bloques. El contraste es más acusado en la zona media central. Este hecho, aunque de forma más moderada -cantos entre 100 y 250 mm.- sucede en la parte distal, formado por coladas más fluidas, de escaso relieve y con una mejor clasificación de los materiales.

En el cauce (perfil 7 C), la distribución es más irregular. El material, poco clasificado -alternan piedras de gran tamaño junto a cantos más pequeños-, varia en función del tipo de depósito en que se incide.

Tambiên se ha representado la evolución de los cantos en los últimos 40 metros del sector distal izquierdo del cono, caracterizado por un material, prácticamente de tipo gravilla, entre 20 y $60 \mathrm{~mm}$. La mayor distancia recorrida por el material justifica ese tamaño sensiblemente inferior al de la zona del ápice.

\subsection{Relación del tamaño de cantos con otros parámetros}

a) El tamaño medio de los cantos en cada punto de muestreo ha sido relacionado con la distancia recorrida desde el ápice y con la pendiente. Para la primera, la correlación lineal es muy baja $(r=<0.300)$ en ambos conos. Los valores eran prácticamente despreciables cuando la relación se hacía con pendiente: $r=0.097$ para el cono de Pitare; estos indices indican que son otros los factores que influyen en la distribución superficial del tamaño de cantos.

b) Tamaño medio de las piedras más gruesas y pendiente (Figura 8). Cuando ambos parámetros fueron relacionados se observaron una serie de pautas en su comportamiento:

-El gráfico de Pitare (1) muestra tres nubes de puntos bien delimitadas que corresponden a sectores definidos anteriormente, con dos tendencias claras:

1. Para pendientes similares, incluso inferiores a las del resto del conjunto del cono, es el cauce (a) con una dinámica muy torrencial, el que exhibe los cantos más gruesos (más de $700 \mathrm{~mm}$ ). El tamaño, aunque no la pendiente, disminuye bruscamente en las márgenes derecha e izquierda del cono (c), es decir, en aquellos puntos donde las aguas, todavia rápidas pero menos violentas que en la primera fase de la inundación y deposición han dejado sedimentos más pequeños (<250 mm.). Las zonas intermedias y distales (b), más disectadas, muestran, para iguales pendientes a las anteriores, tamaños intermedios. 


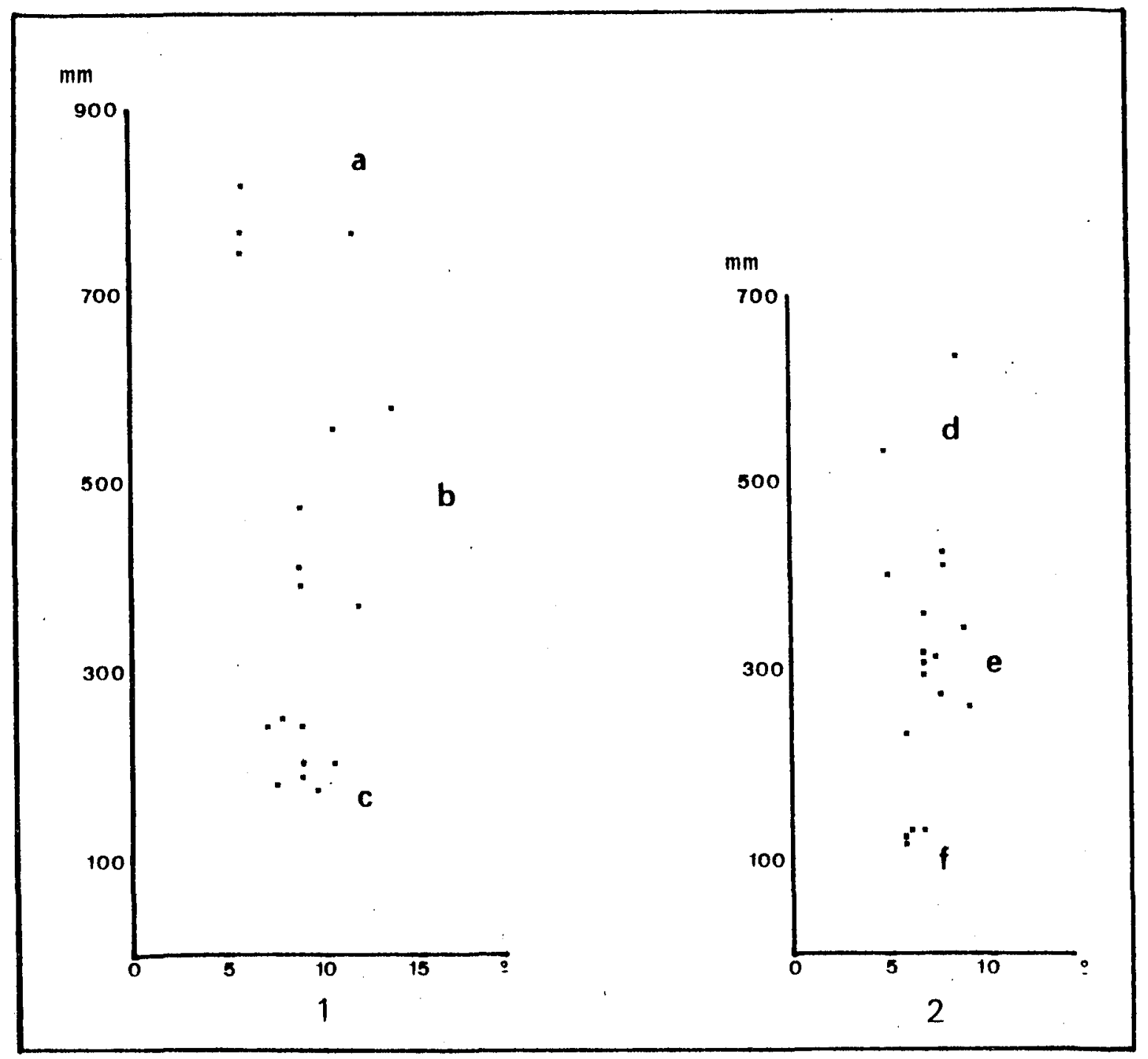

Flg. 8. Relación del tamaño medio de los cantos y el coeficiente de variación. 1. Cono de Pitare. 2. Cono de Banadero.

2. Es en estos dos sectores donde se aprecia bien una disminución de competencia de los flujos deposicionales y, por lo tanto, de cantos gruesos con la reducción de la pendiente.

-En el cono de Banadero varía ligeramente la competencia de los modelos deposicionales que formaron el cono. Con un intervalo de pendientes mucho más ajustado desde el ápice a la base (entre $6^{\circ}$ y $9^{\circ}$ ), son las coladas de piedras (d) de la zona central las que arrastran los cantos más groseros (500-600 mm.). En el sector del ápice y distal izquierdo (f), formados en las fases finales de la deposición, los clastos más gruesos disminuyen a medida que lo hace la pendiente. Para los puntos muestreados en el resto de las coladas y en los canales (e), los valores de tamaños son intermedios y la tendencia es menos clara. 
GOMEZ-VILLAR

\section{Discusión}

La sedimentologia de los depósitos de conos aluviales es muy compleja debido a los numerosos procesos que intervienen en su formación. La respuesta de la cuenca de drenaje causa, además, un aumento en su variabilidad (SCHUMM, MOSLEY \& WEAVER, 1987). Los fuertes aguaceros juegan un importante papel en la movilización de sedimentos en las áreas fuente transportándolos dentro del sistema fluvial (KELSEY, 1970; PIERSON, 1986) y sobre la superficie de los conos (HARVEY, 1989; Wells \& HARVEY, 1987). Durante el transporte, la proporción de agua/sedimento puede variar y la transformación de coladas de piedras a procesos fluviales puede ocurrir cono abajo (LAWSON, 1971; PIERSON \& SCOTT, 1985 y, en consecuencia, generar diversas tendencias en la distribución superficial de los sedimentos.

De los análisis llevados a cabo en los conos de Pitare y Banadero se desprenden una serie de pautas en la distribución del tamaño de cantos muy significativas:

Cono de Pitare:

-Aumento del tamaño de los cantos de "fuera a dentro", es decir, desde la superficie, no incidida, hacia la zona del canal funcional actual. Los perfiles topográficos transversales reflejaban los diferentes niveles de disección, de manera que a cada nivel de incisión le corresponde un aumento del tamaño medio de los cantos.

-Aumento del tamaño desde el apice a la base en el canal y en el sector central, la zona más disectada de todo el cono.

-Disminución del tamaño de cantos desde el ápice a la base en los sectores más alejados del canal, que no han sido afectados por flujos posteriores a la deposición del cono.

Cono de Banadero. No se puede hablar de tendencias, aunque podemos distinguir varios sectores:

-Los cantos más gruesos se localizan en el sector central de la zona media del cono. El calibre de los cantos en la zona media distal, aunque ligeramente inferior, también es importante. Ambos sectores corresponden aun complejo deposicional a base de coladas de piedras.

-Una gran homometría y un menor calibre medio se da en el sector del ápice y distal izquierdo, ambos correspondientes a la última fase de deposición, más fluvial, del cono.

-Los cantos de los canales no muestran una tendencia definida y su distribución está en función de las caracteristicas sedimentológicas de los distintos sectores en los que incide.

La tendencia normal del tamaño de los sedimentos sobre la superficie del cono es la disminución del calibre como abajo -BLISSENBACH, 1952; BuLL, 1964, SCHUMM, MOSLEY \& WEAVER, 1987-, incluso de forma exponencial con la pendien- 
te (KRUMBEIN, 1935). Sin embargo, tal como se ha pretendido explicar para los conos de Pitare y Banadero, la evolución de sus características sedimentológicas no responde a estos parámetros. Asi se ha podido observar en las relaciones establecidas entre la distancia y pendiente con el tamaño medio de los cantos. BEAUMONT (1972) indica que existe poca correlación en la reducción del tamaño de los cantos con la pendiente y no presenta una tendencia bien marcada cuando se trata de materiales groseros (Figura 8). El tamaño medio de las piedras más grandes varia de 119 a $800 \mathrm{~mm}$. en Pitare, en una pendiente de $6^{\circ}$ a $12^{\circ}$, y de 77 a $460 \mathrm{~mm}$. en Banadero, con pendientes entre $6^{\circ}$ y $9^{\circ}$. Estos resultados, comparados con los obtenidos por LUSTIG (1965) en California, posiblemente reflejen el tamaño más pequeño de estos conos.

La dinámica y características de los propios mecanismos que han generado los conos explican la distribución de los sedimentos en superficie, así como la influencia de los factores topográficos y climáticos en la producción de sedimentos dentro de sus cuencas.

Las grandes proporciones de sedimentos groseros que accedieron a estos conos están directamente asociadas con las fuertes precipitaciones que dieron lugar a su formación (HARVEY, 1989). En el caso de Pitare, la ausencia de una cubierta vegetal densa -debilitada tras un incendio- y una densidad de drenaje elevada (3.7) favorecieron la râpida generación de la escorrentía y la llegada de una alta proporción de sedimentos hacia el cauce principal, por barrido de los sedimentos acumulados en los canales seguidos de una labor de socavamiento de la parte baja de las vertientes, con gran aporte de finos (ARNAEz et al, 1987).

Durante la tormenta, muchos de estos materiales, removidos del fondo del cauce, son depositados sobre la base del cono en forma de coladas de piedras. Posiblemente el puente situado sobre el barranco, próximo a su desembocadura contribuyó a frenar el avance de la totalidad de la carga al río Najerilla, depositándose inmediatamente aguas arriba del puente, en forma de cuña, con un mayor espesor en los bordes del cono. La elevada energía del flujo y el gran contenido en finos en este primer impulso de remoción de sedimentos permitió el desplazamiento de los cantos más gruesos hasta la zona distal. Inmediatamente por encima de este sector se depositaron coladas de piedras más fluídas (HARVEY, comunicación personal), dando lugar a una graduación del calibre del sedimento, con cantos menos gruesos hacia el ápice. Estas coladas, en el sector central, se hallan a distinto nivel aunque con una diferencia de minutos en su deposición, ya que corresponden a un único suceso tormentoso.

Todo el conjunto estaba cubierto por material más fino y clasificado en superficie. Una corriente de aguas más claras, probablemente distribuyó bien estos sedimentos en la superficie del cono en los últimos pulsos de la crecida y causó un enterramiento parcial de las coladas en las partes más bajas. La gran disección a que fue sometido durante e inmediatamente después de su formación, presentando niveles bien marcados, tal como revelan los perfiles transversales, permitió observar la morfologia y justificar la localización de los sedimentos más gruesos en las coladas de los sectores central y distal (Foto 2) y en el canal central, que incide sobre las mismas. 
En superficie, el sector izquierdo se caracteriza por formas lobuladas, con márgenes bien definidas (Foto 3). GARCIA-RuIZ et al, 1988, indican que este estadio coincide con el colapsamiento de algunos laterales del cauce, tras una labor de zapa en las orillas con arrastre de cantos de mediano y pequeño calibre. Estas coladas se detienen al llegar al cono y no lo cubren del todo, disminuyendo en tamaño y espesor hacia el ápice. El sector derecho parece corresponder a una fase más fluvial, de aguas menos violentas, caracterizado por un material más pequeño y homométrico. Estos cambios graduales en el tamaño de gravas sugieren un decrecimiento de competencia durante las últimas etapas de inundación; asimismo, la continuidad del canal que corta todos los sedimentos sugiere una reducción en la disponibilidad de sedimento. La secuencia deposicional de coladas de piedras formaron los distintos niveles seguidos por fases más diluídas hasta que ocurrió la sedimentación fluvial.

La situación es distinta para el cono del Banadero: las laderas estabilizadas, cubiertas por un espeso hayedo y la presencia de una cuenca de recepción activa en su cabecera como principal fuente de sedimentos permitieron la intervención de otros mecanismos, de carácter más fluvial, en su formación.

Los sedimentos se movieron aguas abajo del canal desbordándose sobre una cascada de sustrato rocoso en el ápice del cono. Aunque la turbulencia pudo tener lugar en la cascada, en los primeros momentos de la tormenta, el agua

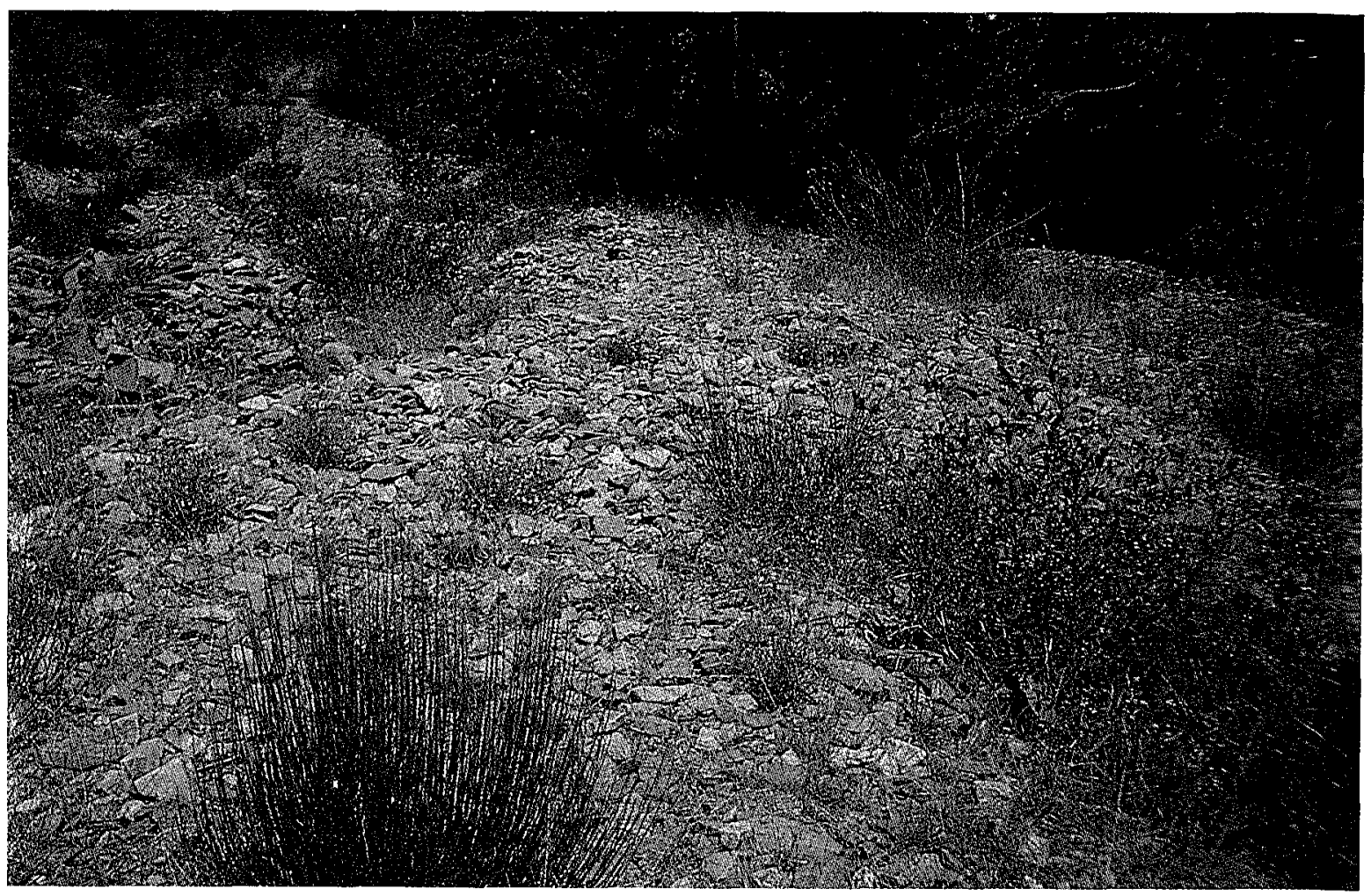

Foto 3. Formas lobuladas, con márgenes bien definidas en el sector "izquierdo" del cono de Pitare. 
retomó los sedimentos aguas abajo de la misma y generó un complejo sisterma deposicional a base de coladas de piedras en la zona central, con material muy heterométrico. La presencia de árboles -que ha semienterrado el cono- en este sector (Foto 4) impidió el avance de este flujo inicial y favoreció su deposición aguas arriba de los mismos. Esta secuencia fué seguida por otras fases más diluídas hacia la zona distal, con cantos más pequeños, puesto que los sedimentos más gruesos quedaron retenidos entre los árboles.

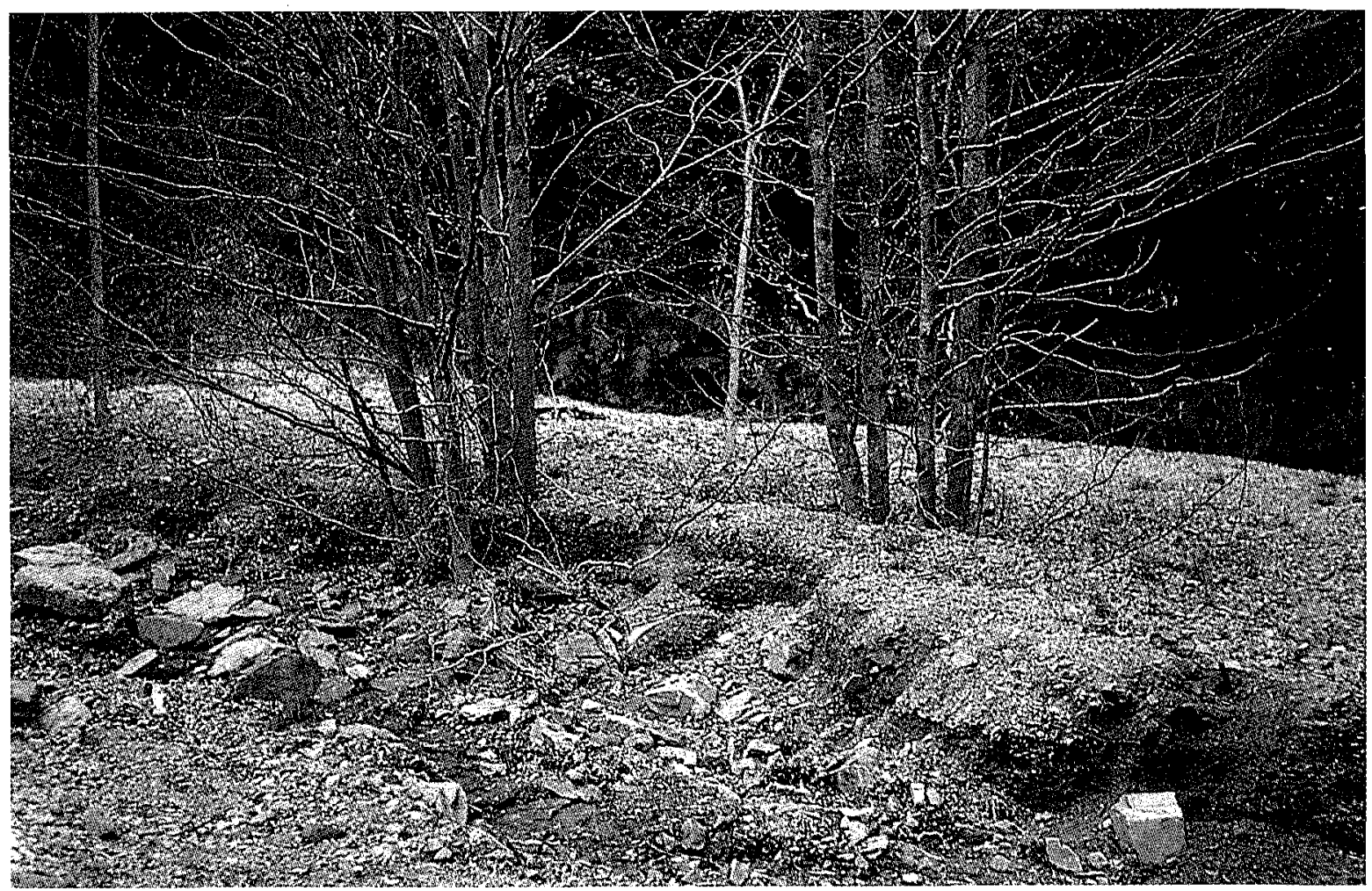

Foto 4. Arboles semlenterrados en el sector central del cono de Banadero.

Durante la tormenta, los flujos fueron cambiando de coladas de piedras a otros de tipo fluvial -caracterizados por un material muy bien clasificado y de tamaño sensiblemente inferior a los anteriores- en el ápice (Foto 5), en los últimos momentos de expansión del flujo. La margen izquierda, en la parte distal fue también creada por deposición fluvial, en un tiempo no superior a 15 minutos (HARVEY, comunicación personal). El agua retomó parte de los materiales finos de las coladas en el sector central y los depositó en este sector caracterizado por un material suelto y muy homométrico. Este hecho coincide con lo observado por BEATY (1970), quien asegura que gran parte del material 
de coladas de piedras es retrabajado por procesos fluviales que ocurren después, pero en la misma tormenta. La disección posterior por canales a ambos lados del sector central deja ver las caracteristicas sedimentológicas de cada sector que incide y de ahí la mayor variabilidad de tamaños de cantos en los mismos.

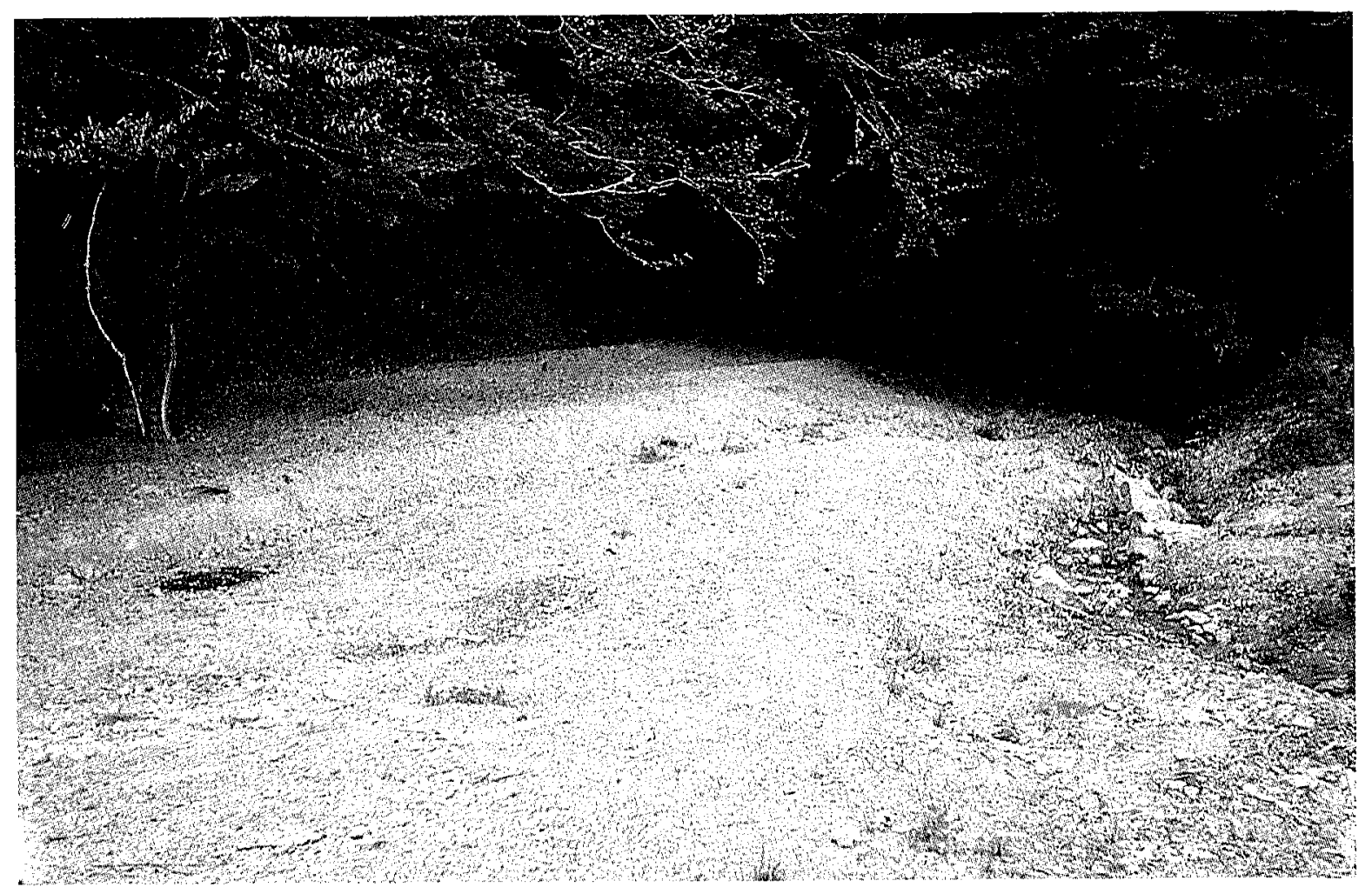

Foto 5. Deposiciôn de típo fluvial en áplce del cono de Banadero.

Las características de la distribución de los cantos sugiere que la velocidad fue relativamente baja y que las condiciones de flujo fueron turbulentas hasta derivar en condiciones más fluviales desarrolladas al final de la tormenta.

Finalmente, la competencia de los flujos que crearon ambos conos queda reflejada en la relación entre el coeficiente de variación y el tamaño medio de los cantos (Figura 9). La correlación es significativa en el cono de Pitare $(r=0.841)$, y desciende notablemente en el de Banadero. Son los depósitos fluviales, con el material más homométrico y de menor calibre, distribuído en pocos intervalos de frecuencias, los que presentan tamaños de cantos y coeficientes de variación más bajos; a su vez, son las coladas de piedras y el canal funcional en el caso de Pitare, los que tienen los tamaños medios y coeficiente de variación más elevados. La variabilidad de la distribución granulométrica de las coladas de piedras se explica por el propio mecanismo del movimiento: se trata un medio viscoso con material fino que facilita el arrastre a su vez de grandes bloques. Es 
TAMAÑO DE SEDIMENTOS EN CONOS ALUVIALES
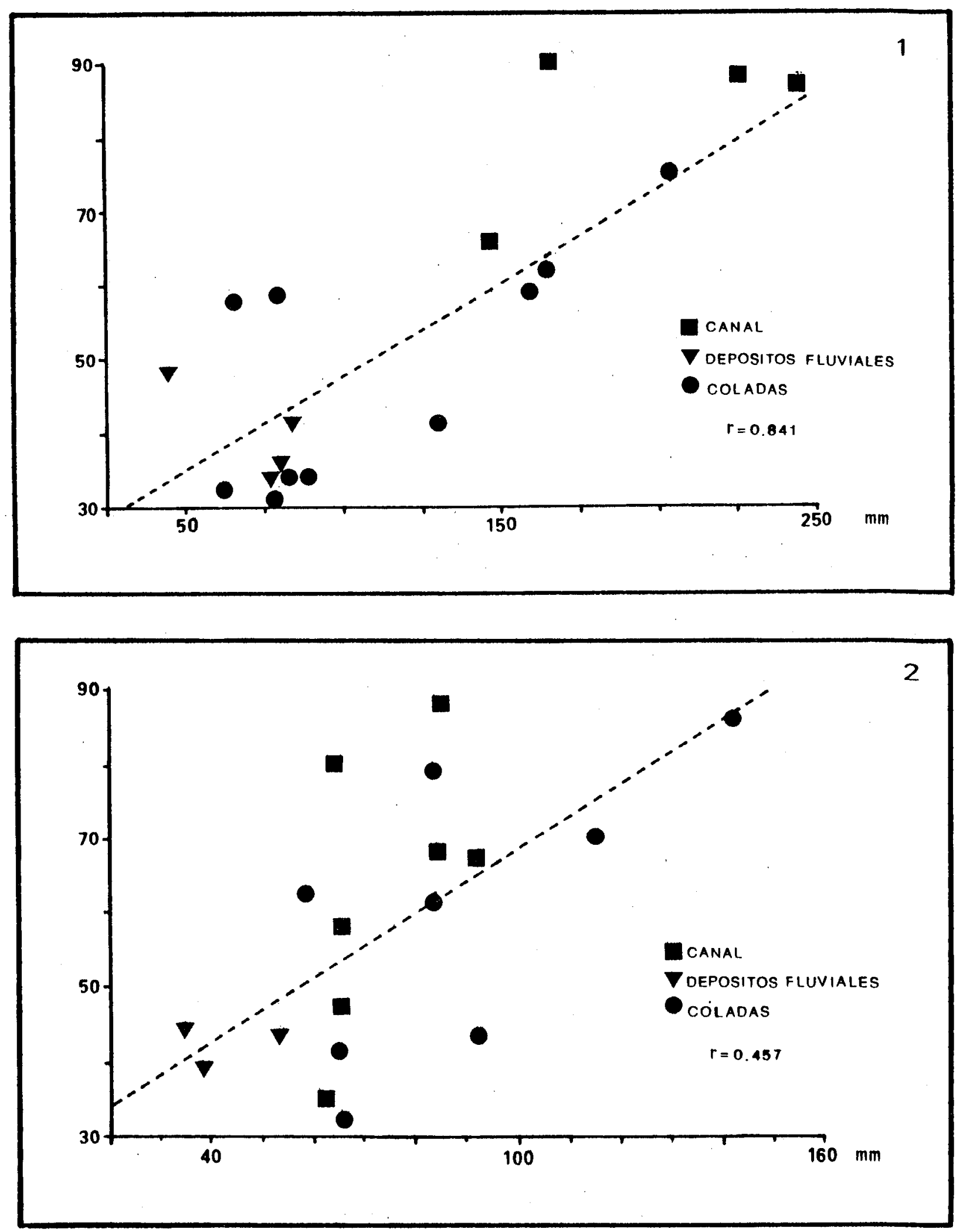

Fig. 9. Relación del tamaño medio de los cantos y el coeficiente de varlación.

1. Cono de Pitare. 2. Cono de Banadero. 
GOMEZ-VILLAR

evidente que los mecanismos que intervienen en la formación de un cono son los principales agentes controladores de su morfologia y distribución espacial superficial de los sedimentos.

Agradecimientos. Este trabajo se ha elaborado dentro del proyecto: "Dinámica de cauces en el Pirineo Central”, como aportación del CSIC al Proyecto LUCDEME.

\section{Bibliografia}

ARNAEZ,J., 1984. Microformas de erosión y acumulación en pistas forestales: el ejemplo del Alto Valle del Cárdenas (Sierra de la Demanda). I Coloquio sobre procesos actuales en Gomorfologia. Cuadernos de Investigación Geográfica, X (1-2): 7-15.

ARNAEZ, J., 1987. Formas y procesos en la evolución de vertientes de la Sierra de la Demanda. Cuadernos de Investigación Geográfica, XIII (1-2): 153 pp. Logroño.

ARnaez, J., y Garcia-Ruiz, J.M. 1984. Tipo de escorrentia y ritmo de evacuación de sedimentos en la Sierra de la Demanda (Sistema Ibérico). I Coloquio sobre procesos actuales en Geomorfologia. Cuadernos de Investigación Geográfica, X (1-2): 17-28. Logroño.

ARnaez, J., Garcia-Ruiz, J.M., Gómez-VillaR, A., y OrTigosa, L.M., 1987. Incendio forestal y degradación del suelo: movimientos en masa en el Alto Valle del Najerilla. Informe para la Consejería de Ordenación del Territorio y Medio Ambiente. Comunidad Autónoma de La Rioja. 56 pp. Inédito.

BAKER, V.R., 1977. Stream channel response to floods with examples from central Texas. Geological Survey of America Bulletin, 88: 1057-1071.

BEATY,CH.B., 1974. Debris flows, alluvial fans and revitalized catastrophism. Zeitschrift für Geomorphologie. Suppl. Bd., 21: 39-51.

BEAumont, P., 1972. Alluvial fans along the Foothills of Elburz Mountains Iran. Paleogeography, Paleoclimatology, Paleoecology. Elsevier Publishing Company Amsterdan, 12: 251-277.

BLISSENBACH, E., 1952. Relation of surface angle distribution to particle size distribution on alluvial fans. Journal of Sedimentary Petrology, 22 (1): 25-28.

BOOTHROYD, J.C., and NUMMEDAL, P., 1978. Proglacial braided outwash a model for humid alluvial fan deposits. In: Miall, A.D. (Ed.). Fluvial Sedimentology: Canadian Society of Petroleum Geologist Memoir, 5: 641-668.

CAINE, N., 1980. The rainfall intensity, duration control of shallow landslides and debris flows: Geografiska Annaler, 62 A: 23-27.

CALVo, J.L., 1975. Nota sobre las relaciones de la red fluvial camerana y la tectónica del borde septentrional del Sistema Ibérico. Berceo 88: 93-99.

COSTA, J.F., and JARRET, R.D., 1984. The 1982 Lawn Lake and Cascada Lake dam fissures Boulder, Colorado. Guidebook to Field trip. 96 pp.

García-Ruiz, J.M., ARnaez, J., Gómez-Villar, A., y OrTigosa, L.M., 1988. Debris flows subsequent to forest fire in the Najerilla river valley (Iberian System, Spain). Pirineos: 3-24.

HARVEY, A.M., 1984. Debris flows and fluvial deposits in Spanish Quaternary alluvial fans: Implications for fan morphology. In: KOSTER, E.H. and STEEL, R.J. (EDS). Sedimentology of gravels and conglomerates. Canadian Society of Petroleum Geologist Memoir, 10: 123134. 
HARVEY, A.M., 1989. The ocurrence and role of arid zone alluvial fans. Arid zone geomorphology. THOMAS, D.S.G. (Ed), pp: 136-158.

Iso, N., Yamakawa, K., Yonezawa, H. and Matsubara, T., 1970. Acumulation rates of alluvial cones constructed by debris flows deposits in the drainage basin of the Takahara River, Gifu Prefecture, central Japan. Geological Review of Japan, 53: 669-720.

KELSEY, H.M., 1980. A sediment budget and analisis of geomorphic process in the $V$ an Duren River Basin, north coastal California 1941-1975: Summary. Geological Society of America Bulletin, 91: 190-195.

KRUMBEIN, W.C., 1937. Sediments and exponential curves. Journal of Geology, XLV: 577 604.

LAWSON, D.E., 1981. Movilization, movement and deposition of active subaerial sediments flows. Matanuska Glacier, Alaska. Journal of Geology, 90: 279-300.

LUSTIG, L.K., 1965. Clastic sedimentation on Deep Spring Valley, California. U.S. Geological Survey Proffesional Paper, 352 F: 131-192.

Newson, M., 1980. The geomorphological effectiveness of floods. a contribution stimulated by two recents events in mid-Wales. Earth Surface Processes, 5: 227-247.

Pierson, T.C., 1980. Erosion and deposition by debris flows at Mt. Thomas, North Canterbury, New Zealand. Earth Surface Processes, 5: 227-247.

PiERSON,T.C., and SCOTT, K.M., 1985. Downstream dilution of a lahar: transition from debris flows to hyperconcentrated streamflow". Water Resources Research 21: 1511-1524.

SChumm, S.A., Mosley, M.P. \& WEAVER, W.E., 1987. Experimental Fluvial Geomorphology. John Wiley \& sons. 413 pp.

TUNBRIDGE, I.P., 1983. Alluvial fan sedimentation of the Horseshoe Park flood, Colorado, USA, July, 15 th, 1982. Sedimentary Geology, 36 (1): 15-23.

WASSON, R.J., 1977. Catchment processes and the evolution of alluvial fans in the lower Derwent valley, Tasmania. Zeitschrifk für Geomorphologie, 21: 147-168.

WeLIS, S.G., and HARVEY, A.M., 1987. Sedimentologic and geomorphic variations in storm generated alluvial fans, Howgill Fells, Northwest England. Geological Society of America Bulletin, 98: 178-198.

Wollman, M.G., and Miller, J.C., 1960. Magnitud and frecuence of forces in geomorphic processes. Journal of Geology, 68: 54-74. 
